\title{
On Parallelized Serially Concatenated Codes
}

\author{
Orhan Gazi and A. Özgür Yılmaz
}

\begin{abstract}
Serial concatenated codes show very good performance at low signal to noise ratios. However, large decoding delays due to long input frame lengths constitute a major disadvantage for these type of codes. In this study we introduce a new class of concatenated convolutional codes which are very suitable for parallel decoding, thus have much less decoding delays and also show comparable performance to that of classical serial concatenated convolutional codes. The analytical upper bound expressions for performance of the proposed structures are derived using the uniform interleaver concept.
\end{abstract}

\section{INTRODUCTION}

$\mathbf{C}$ ONCATENATED codes are proposed by Forney [1] in 1966. The usage of a posteriori probabilities (APPS) as opposed to hard decision estimates is essential for enhancing decoder performance. Following Forney's work, new APP algorithms were developed. The BCJR algorithm [2] which was ignored for a long time is one of the most widely known APP algorithms. With the introduction of turbo codes (parallel concatenated convolutional codes, i.e., PCCCs) [3] in 1993 a huge interest on iterative decoding was aroused. The use of soft-in soft-out decoding algorithms was a key in the astonishing performance of turbo codes. This showed the way to the invention of serially concatenated convolutional codes SCCCs in [4], where authors showed that they were better than PCCCs in some respects. In the last decade, similar codes such as, low density parity check codes [5], block product codes [6], and single parity check (SPC) product codes [7] have been extensively studied.

Convolutional codes have some appealing properties over linear block codes. Convolutional codes' trellis structure does not vary in time as in block codes. Moreover, the number of states in the trellis structure of an $(n, k)$ block code is upper bounded by and usually on the order of $2^{(n-k)}$ where $k$ is the information sequence length and $n$ is the codeword length in bits [8]. However, the number of states in a convolutional code can be set as desired. The time invariant trellis structure of convolutional codes makes them more convenient for implementation [4]. In addition, numerous practical techniques such as trellis coded modulation (TCM) and puncturing can be easily utilized with convolutional codes, as opposed to linear block codes.

Concatenated convolutional codes $C C C s$ are potential candidates for practical applications in many communication systems. Low latency is necessary if high data rates are

Orhan Gazi is with Electronics and Communication Engineering Department, Cankaya University, Ankara, Turkiye. o.gazi@cankaya.edu.tr

A. Ozgür Yılmaz is with Electrical and Electronics Engineering Department, Middle East Technical University, Ankara, Turkiye. aoyilmaz@eee.metu.edu.tr required. Since CCCs are iteratively decoded, the decoding delay may not be affordable for high data rate communication systems. One possible solution is to implement $C C C$ decoders in a parallel fashion. In [9], [10], [11] techniques for parallel realization of turbo decoders were suggested. The common idea of these studies is to divide the received information block into sub-blocks and decode the sub-blocks in a parallel manner. However some difficulties arise in these applications. In [10] and [11] trellis blocks are divided into overlapped sub-blocks and these sub-blocks are decoded using the same soft-in soft-out (T-Algorithm or MAP) decoders which utilizes only partial observations. Thus, the method in [10] and [11] is sub-optimal unless a reasonable overlapping depth is used. A similar method was introduced in [9] where forward and backward variables computed in the previous iteration are used to provide boundary distributions for each block sub-block MAP decoders. This method requires additional memory in hardware to store the boundary distributions.

Turbo product codes based on convolutional codes are studied in detail in [12] where parallelization is applied at the encoder side so that parallel processing at the receiver is directly available without adding any extra algorithms. The receiver side directly employs $M A P$ decoders in parallel. No overlapping, no boundary distributions or any other extra considerations are needed at the receiver side. We extend the studies in [12] with this paper and propose general structures for serially and parallel concatenated codes. SCCCS and $P C C C s$ are in fact special cases of the proposed structures. We show that PDSCCCs can be used instead of the SCCCs considerably reducing decoding delays.

The outline of the paper is as follows. In Section II definitions of weight spectrum functions are specified and analytical upper bound expressions are given for concatenated codes using the uniform interleaver concept. In Section III the proposed code configurations are explained. The advantage of the proposed codes is mentioned in Section IV. Simulation and analytical analysis results are given in Sections V and VI. Finally, concluding remarks are given in Section VII.

\section{BACKGROUND}

Analytical bound expressions for the performance of serial and parallel concatenated codes are defined in [4]-[13] where the authors employ uniform interleaving which is defined in [13] as a probabilistic device that maps a given input information sequence of length $K$ and Hamming weight $w$ into all distinct $\left(\begin{array}{l}K \\ w\end{array}\right)$ permutations with equal probability $1 /\left(\begin{array}{l}K \\ w\end{array}\right)$. The uniform interleaver permits the estimation of 
the average interleaver gain, independent of the particular interleaver used in a concatenated code system.

\section{A. Serially Concatenated Codes}

The encoder for serially concatenated codes (SCCs) is depicted in Fig. 1. The constituent codes can be chosen from block and convolutional codes. If both constituent codes are chosen from convolutional codes, the well known serially concatenated convolutional codes SCCCs are obtained.

Although two constituent encoders are employed in Fig. 1, the idea can be extended to any number of constituent encoders by incorporating more encoders and interleavers.

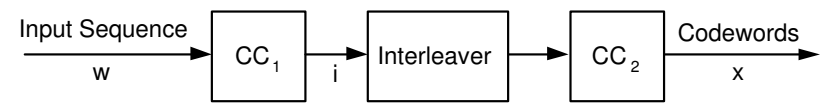

Fig. 1. Serial concatenated code. $C C_{1}$ and $C C_{2}$ are constituent codes CCs 1 and 2, respectively. The interleaver size is $\mathrm{N}$.

\section{B. Analytical Analysis of SCCCs}

1) Conditional Weight Enumerating Function (CWEF): The conditional weight distribution function gives us information about the weight distribution of codeword sequences or parity sequences generated from a Hamming weight $w$ input sequences:

$$
A(w, X)=\sum_{i} A_{w, i} X^{i}
$$

where $i$ is the Hamming weight of the codewords generated by input sequences of Hamming weight $w$.

2) Input Output Weight Enumerating Function (IOWEF): IOWEF contains information about the Hamming weight of the input sequences along with the produced codewords:

$$
A(W, X)=\sum_{w, i} A_{w, i} W^{w} X^{i}
$$

where $A_{w, i}$ is the number of codewords of Hamming weight $i$ generated by information sequences of Hamming weight $w$. $W$ and $X$ are dummy variables.

3) IOWEF of SCCCs: Referring to Fig. 1, IOWEF of serial concatenated code $A^{C_{s}}(W, X)$ is expressed as a product of the two CWEFs of the constituent codes, which is normalized by the number of the possible permutations (i.e., uniform interleaver)

$$
A^{C_{s}}(W, X)=\sum_{i=0}^{N} \frac{A^{C C_{1}}(W, i) \times A^{C C_{2}}(i, X)}{\left(\begin{array}{c}
K \\
i
\end{array}\right)} .
$$

Once IOWEF of a concatenated code is available, upper bounds of the bit error probability can be calculated using (2) and (3).
4) Analytical Bound: Once spectrum functions of the concatenated codes are available, performance upper bound to the bit error probability for maximum likelihood soft decoding of the code is found in the form

$$
P_{b}(e) \leq \sum_{w, i} \frac{w}{2 k} A_{w, i} \operatorname{erfc}\left(\sqrt{\frac{i R_{c} E_{b}}{N_{0}}}\right) .
$$

This is further simplified as [13]

$$
P_{b} \cong \frac{1}{2} \sum_{i} D_{i} \operatorname{erfc}\left(\sqrt{\frac{i R_{c} E_{b}}{N_{o}}}\right),
$$

where $R_{c}$ is the code rate, $\frac{E_{b}}{N_{0}}$ is the bit energy to noise ratio of the $A W G N$ channel, $i$ is the Hamming weight of the codewords generated by Hamming weight $w$ input sequences. For SCCCs $D_{i}$ 's are obtained from the IOWEF coefficients according to

$$
D_{i}=\sum_{w} \frac{w}{k} A_{w, i} .
$$

\section{Parallel Decodable Concatenated Codes}

(PDCCS)

To reduce the decoding delays of turbo-like codes, researches developed some techniques which involve parallel processing in the decoding operation [9], [10], [11]. In these studies, parallelization is considered at the receiver side. In our study we employ the parallelization at the transmitter side and use it directly at the receiver side without requiring any extra algorithms. The parallel encoder structure for SCCCs is depicted in Fig. 2 where $N$ and $M$ are the number of outer and inner constituent encoders for SCCCs. Serial to parallel $(S / P)$ and parallel to serial $(P / S)$ converters are employed.

\section{A. Parallel Decodable Serially Concatenated Codes (PDSCCs)}

The general structure of the PDSCCs is depicted in Fig. 2 where $C_{11} \ldots C_{1 N}$ and $C_{21} \ldots C_{2 M}$ are the outer and

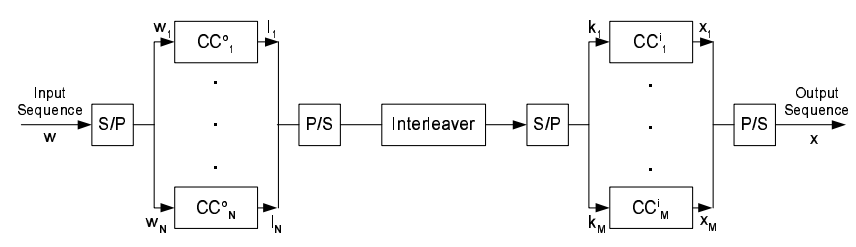

Fig. 2. $\quad P D$-SCC model. $C C_{n}^{o}, n=1 \ldots N$ and $C C_{m}^{i}, m=1 \ldots M$ are constituent encoders.

inner constituent code $(C C)$ encoders respectively. $N$ and $M$ are the number of constituent encoders in the outer and inner clusters, respectively. An interleaver is placed between the inner and outer encoder clusters. If all the constituent codes are chosen from the same linear block code and a rectangular interleaver is employed between code clusters, the well known block product codes are obtained. Block product codes are usually expressed using a matrix notation for ease of illustration. The matrix equivalent representation for the block product code is depicted in Fig. 3. 


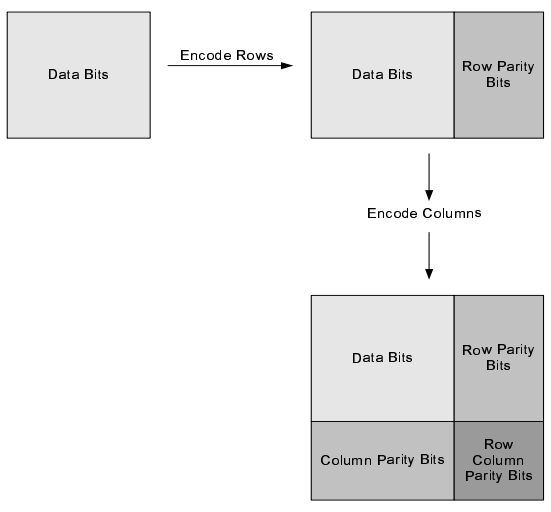

Fig. 3. Regular product code encoding procedure, where a block code is used to encode rows and columns.

1) Parallel Decodable Serially Concatenated Convolutional Codes (PDSCCCS): If convolutional code encoders are employed in PDSCCs, PDSCCCs are obtained. When $M=N=1$ PDSCCCs turn out to be SCCCs, i.e., SCCCs are a special case of PDSCCCs. Assuming that the rate of all the constituent encoders equals $1 / 2$, for an input sequence of length $(N \times M) / 2$ where $N \geq 2$ and $M=2 k, k$ a positive integer, the overall encoding procedure can be demonstrated using a matrix notation. This special case will be named as matrix PDSCCCs (i.e., M-PDSCCCs). If $M$ value is chosen as $2 N$ then the input matrix becomes a square matrix. The encoding procedure is depicted in Fig. 4. We didn't show the addition of trellis termination bits to the encoded data in the Fig. 4, however the effects of trellis termination bits are taken into account in simulations. The length of the input subsequences of the outer and inner $C C$ encoders equals $M / 2$ and $N$ respectively. The interleaver size is $N \times M$. Although a rectangular interleaver is employed between constituent encoder clusters for ease of illustration any type of interleaver can be used. We will use a square input matrix $(M=2 N)$ in our simulations. The encoding operation using the matrix

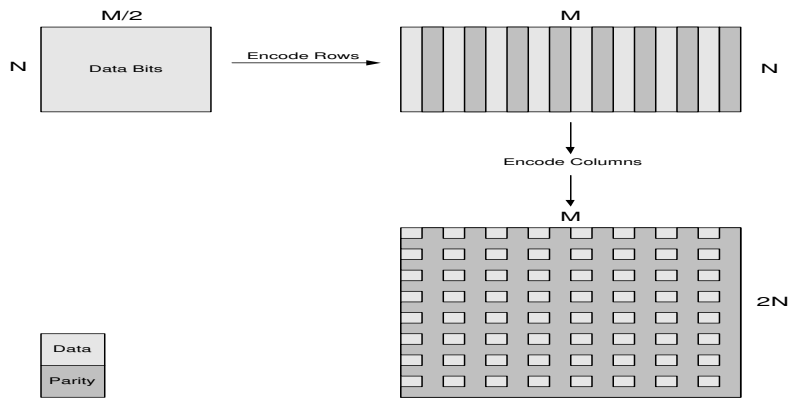

Fig. 4. M-PDSCCC encoding procedure.

structure proceeds as follows. The data to be sent is put into a matrix. Each row of the matrix is encoded using a code encoder. Once all the rows are encoded, the matrix is sent to an interleaver. The interleaved matrix is coded columnwise. Data matrix dimension is $k x k$, and encoded data matrix dimension is $n x n$, i.e., our code is an $(n x n, k x k)$ code.
2) PDSCCC Decoder: The decoder of a PDSCCC is seen in Fig. 5. It is clear from the decoder structure that decoders $D_{11} \ldots D_{1 N}$ or decoders $D_{21} \ldots D_{2 M}$ can run in parallel.

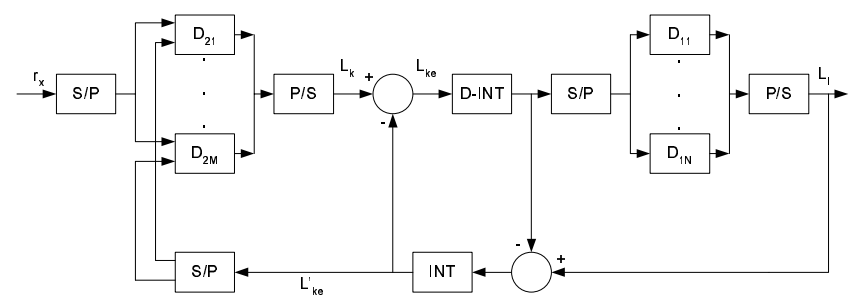

Fig. 5. $P D$-SCCC Decoder. $r_{x}$ is the received signal value. INT, D-INT are the interleaver and de-interleaver. $L_{k}$ and $L_{l}$ are the bit probabilities. $L_{k e}$ and $L_{k e}^{\prime}$ are the extrinsic bit probabilities.

\section{PRACTICAL IMPLEMENTATION ADVANTAGES}

Definition: The complexity operator $C(L)$ for the convolutional code $C$ is $C(L)=k \times L$ where k is the number of parameters (number of alfas, betas, gammas) to be computed by the decoder in a single stage of the code trellis, i.e., for an input frame of length $L$ the total computation amount is approximately $k \times L$. $k$ depends on the convolutional code generator matrix.

Definition: The latency operator is $T(L)=t \times L$ where $t$ is the decoding latency for a single stage of the code trellis. $L$ is the length of the input information sequence.

The implementation advantage of M-PDSCCC will be discussed herein with the parameters used in this study. Trellis termination will be neglected in calculation and will not alter the results significantly. In SCCC, for a given transmit data vector of length $L$, two log-MAP decoders are needed. The first decoder has a complexity of $C(2 L)$ and a time delay of $T(2 L)$. The second decoder has a shorter input, thus it has a complexity of $C(L)$ and a time delay of $O(L)$. In total, the complexity is of $C(3 L)$ and the time delay is of $T(3 L)$. An input sequence of length $L$ corresponds to a square matrix of size $\sqrt{L} \times \sqrt{L}$. In $M$-PDSCCC columns (inner code cluster) are decoded first. The use of separate log-MAP decoders for each row and column makes parallel processing operation possible. Each column decoder (inner decoder) has complexity of $C(\sqrt{L})$ and time delay of $T(\sqrt{L})$. Since these decoders are run in parallel, the total column decoding complexity is of $C(2 L)$ but the time delay is of $T(\sqrt{L})$. Similarly, row decoding (outer decoder) has a total complexity of $C(L)$ and time delay of $T(\sqrt{L})$. Although both complexities are the same, $M$-PDSCCCs decrease the decoding delay by factor $T(\sqrt{L})$.

\section{PDSCCC SIMULATION}

We used the recursive systematic convolutional code $R S C$ with the generator $(1,5 / 7)_{\text {octal }}$ for all constituent code encoders in M-PDSCCC. An S-random interleaver $(S=18)$ is employed between clusters. Input information sequence frame length is chosen as 1024 bits. We formed a size $32 \times 32$ information matrix. Thus, $N$ equals 32 and $M$ equals 68 
(i.e trellis termination bits are added after row encoding). The encoded data in matrix form is multiplexed to a single stream and binary phase shift key (BPSK) modulated. The $B P S K$ modulated signal is passed through an additive white Gaussian noise $(A W G N)$ channel with double-sided power spectral density $\frac{N_{0}}{2}$. We used the $\log -M A P$ soft decoding algorithm [3] to iteratively decode the M-PDSCCCs. We used 12 iterations for decoding. We also simulated the serially concatenated convolutional code which is a special case of $M$-PDSCCC with $M=N=1$. The signal-to-noise ratio values given in all the Figs. are normalized with the proper code rates for all scenarios. Non-recursive systematic convolutional codes were also tried and it was seen that their performance is not as good as the M-PDCCC system where $R S C$ encoders are employed.

\section{A. Trellis Termination Effects}

We investigated the effects of trellis termination for three different scenarios. Trellis termination bits are added by the outer $R S C$ encoders (row encoders) $(R-T)$ with rate $\approx$ 0.235 , both outer and inner constituent $R S C$ encoders (row and column encoders) added trellis termination bits to the codeword $(T T)$ with rate $\approx 0.221$, neither outer and inner constituent RSC encoders added trellis termination bits to the codewords $(N o-T T)$ with rate $\approx 0.25$. Although addition of trellis termination bits decreases the code rate, they are critical for good performance of the $M-P D S C C C$ as seen in Fig. 6. Addition of trellis termination bits in turbo or serially concatenated code shows negligible improvement on the code performance [14]. However, without trellis termination the performance of the $M-P D S C C C$ degrades drastically. The addition of trellis termination bits slightly decreases the code rate, and this leads to a small loss in bandwidth efficiency.

The performance graphs are seen in Fig. 6. M-PDSCCC has better performance at very low $\frac{E_{b}}{N_{0}}$ levels when only inner codes are trellis terminated. Its performance is worse at higher $\frac{E_{b}}{N_{0}}$ levels when it is compared to the inner and outer codes trellis terminated case. Though quite close up to $B E R$ $10^{-7}$, SCCC seems to have an error curve of higher slope compared to $T T$ at higher $\frac{E_{b}}{N_{0}}$ values. The analytical bound is evaluated for the $T T$ case using the uniform interleaver approach. However as seen from the Fig. 6 the bound obtained by uniform interleaver concept gives a rough idea about the code performance.

\section{B. Symmetric PDSCCC}

We also simulated the scenario where an equal number of parallel branches are used for the outer and inner clusters. The performance graph is depicted in Fig. 7. It is obvious from the Fig. 7 that the performance of the PDSCCC is slightly better than that of the $S C C C$ at high $\frac{E_{b}}{N_{0}}$ values.

\section{Analytical Analysis of PDSCCC}

The uniform interleaving assumption can be used to evaluate the average performance of the PDSCCCs. Since the information bits of parallel subsequences of inner the and

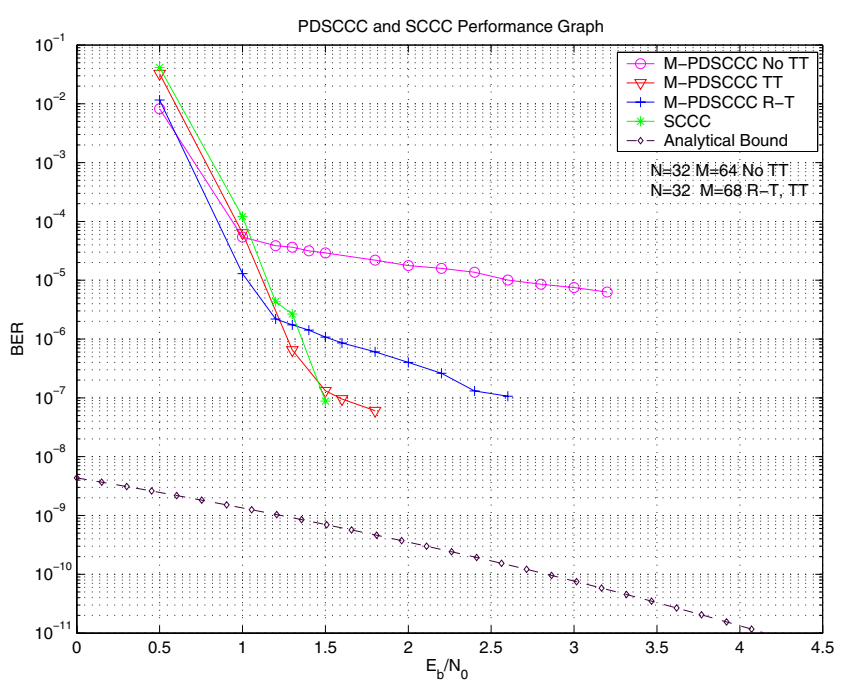

Fig. 6. $M-P D S C C C$ and $S C C C$ performance graph. $M-P D S C C C$ with no trellis termination (M-PDSCCC No TT). M-PDSCCC when rows (outer codes) are trellis terminated (M-PDSCCC R-T). M-PDSCCC when rows and columns (outer and inner codes) are trellis terminated (M-PDSCCC TT). Frame length=1024, Iteration number $=12$.

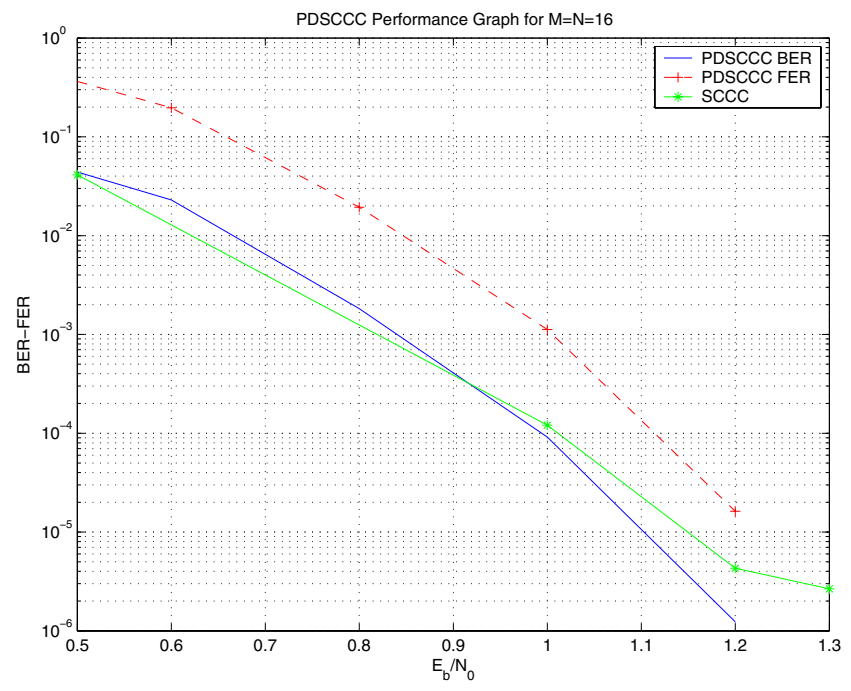

Fig. 7. PDSCCCs performance graph. The number of outer encoder and inner constituent encoders are equal and it is 16 . Trellis termination bits are added by both outer and inner encoders. Frame lenght $=1024$. Iteration number $=12$.

outer clusters are independent, IOWEFs of the inner and outer clusters can be evaluated as

$$
\begin{aligned}
& A^{C 1}(W, X)=\prod_{i=1}^{N} A_{1 i}(W, X) \\
& A^{C 2}(W, X)=\prod_{i=1}^{M} A_{2 i}(W, X),
\end{aligned}
$$

where $A_{1 i}(W, X)$ and $A_{2 i}(W, X)$ are the IOWEFs of the $C C s$ in the inner and outer clusters. We considered different scenarios and evaluated analytical bounds for the performance of the M-PDSCCCs. The analytical bounds for square 
input matrices are shown in Fig. 8 for different interleaver lengths. Trellis termination bits are used by both inner and outer clusters. It is clear from Fig. 8 that as the interleaver size increases better performance is obtained.

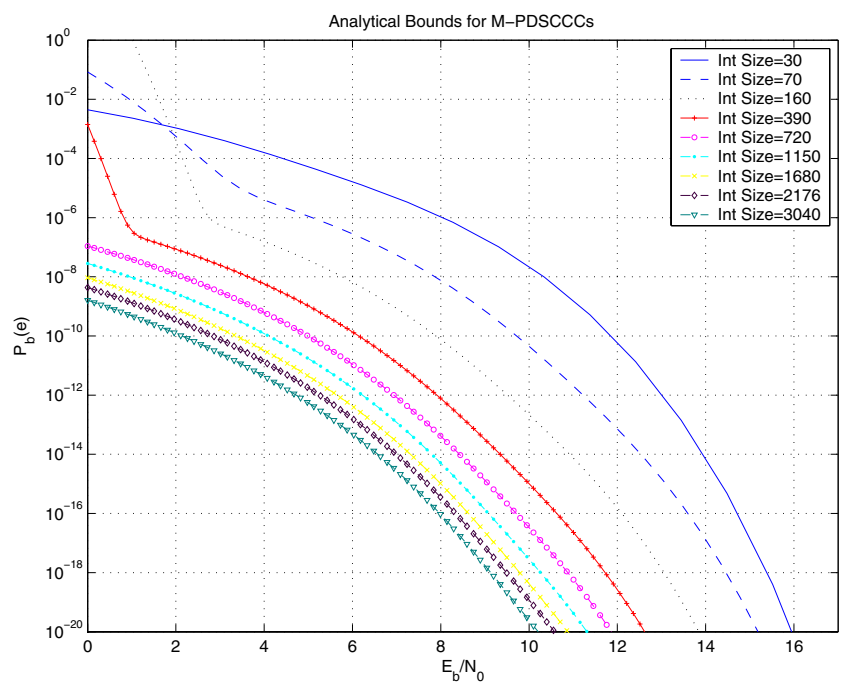

Fig. 8. M-PDSCCCs' analytical bounds for different interleaver sizes. Square input matrices are used.

Analytical bounds are also evaluated for the case when the trellis termination is not applied to the constituent codes. The bounds are shown in Fig. 9. When compared to Fig. 8, the degradation in performance is obvious.

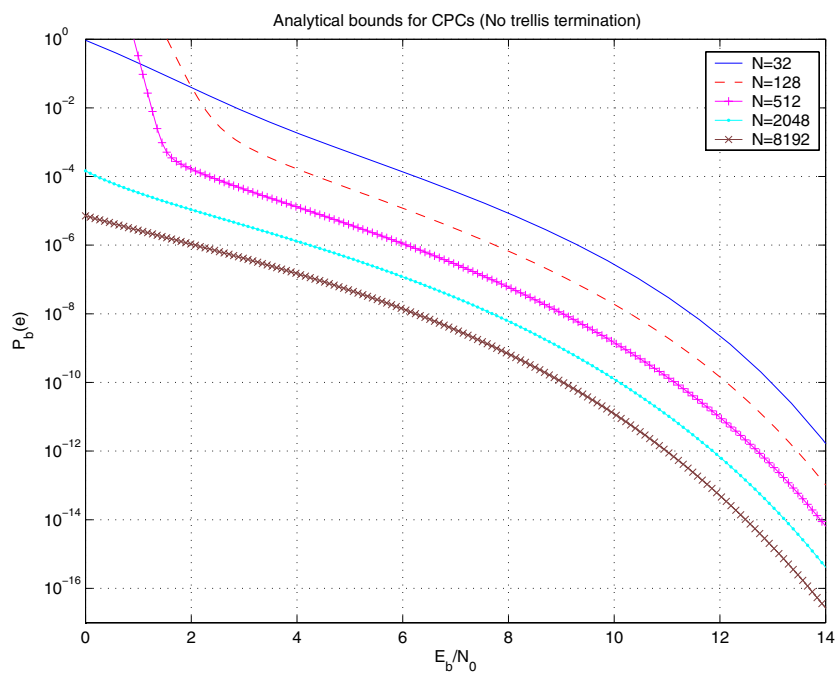

Fig. 9. M-PDSCCCs' analytical bounds when trellis termination bits are not used.

\section{CONCLUSION}

In this article we propose new classes of serial concatenated convolutional codes. Using the uniform interleaver assumption we showed that the proposed codes perform as well as classical serially convolutional codes. Simulation results also support the analytical outcomes. The decoding delay is reduced by a factor of the number of parallel branches. This provides a significant advantage in practical applications.

\section{REFERENCES}

[1] G. D. Forney, Jr., Concatenated Codes, M.I.T. Press, Cambridge, MA, USA, 1966.

[2] L. R. Bahl, J. Cocke, F. Jelinek, and J. Raviv, "Optimal decoding of linear codes for minimizing symbol error rate", IEEE Trans. Inf. Theory, Vol. IT-20, March 1974, pp. 284-287.

[3] C. Berrou, A. Glavieux, and P. Thitimajshima, "Near Shannon limit error-correcting coding and decoding: Turbo-codes," in Proc. ICC'93 (Geneva, Switzerland, May 1993), pp. 1064-1070.

[4] S. Benedetto, D. Divsalar, G. Montorsi and F. Pollara, "Serial concatenation of interleaved codes: Design and performance analysis," IEEE Trans. Inform. Theory, vol. 44, pp. 909-926, May, 1998.

[5] R. G. Gallager, "Low Density Parity Check Codes," IRE Trans. Inform. Theory, IT-8:21-28, January 1962.

[6] P. Elias, "Error free decoding," IRE Trans. Inform. Theory, vol. IT-4, pp. 29-37, Sept., 1954.

[7] D. Rankin, T. Aaron Gulliver, " Single parity check product codes," IEEE Trans. on Comm., vol. 49, no. 8, August 2001 pp. 1354-1362

[8] Hanzo, L. / Liew, T. H. / Yeap, B. L. Turbo Coding, Turbo Equalisation and Space-Time Coding for Transmission over Fading Channels, John Wiley and Sons, 2002.

[9] S. Yoon, Y. Bar-Ness, "A Parallel MAP Algorithm for Low Latency Turbo Decoding", IEEE Communication Letters, Vol. 6, No. 7, July 2002.

[10] U. Dasgupta and K. R. Narayanan, "Parallel Decoding of Turbo Codes Using Soft Output T-Algorithms", IEEE Communication Letters, Vol. 5, No. 8, August 2001.

[11] J. Hsu and C. Wang, "A parallel decoding scheme for turbo codes," in Proc. ISCAS'98, vol. 4, June 1998, pp. 445-448.

[12] O. Gazi, A. Ö. Yılmaz, "Turbo Product Codes Based on Convolutional Codes," ETRI Journal, Vol: 28, No: 4 pp. 453-460, August 2006.

[13] S. Benedetto, G. Montorsi, Unveiling Turbo Codes: Some Results on Parallel Concatenated Coding Schemes, IEEE Trans. on Inform. Theory, Vol. 42, No. 2, March 1996.

[14] P. Robertson, "Illuminating the structure of parallel concatenated recursive (TURBO) codes, " in Proc. GLOBECOM'94, San Francisco, CA, Nov. 1994, pp. 1298-1303. 